\title{
BMJ Open Implementation of a virtual international cardiology curriculum to address the deficit of cardiovascular education in Haiti: a pilot study
}

\author{
Norrisa Adrianna Haynes (D) , ${ }^{1}$ Veauthyelau Saint-Joy, ${ }^{2}$ JaBaris Swain, ${ }^{3}$ \\ Agnes Ezekwesili, ${ }^{4}$ Fritz Verly Vernet, ${ }^{5}$ Calixte Dawson, ${ }^{5}$ Davidson Laneau, ${ }^{5}$ \\ Ann Tierney, ${ }^{6}$ Judy A Shea, ${ }^{7}$ Marietta S Ambrose ${ }^{1}$
}

To cite: Haynes NA, SaintJoy V, Swain JB, et al. Implementation of a virtual international cardiology curriculum to address the deficit of cardiovascular education in Haiti: a pilot study. BMJ Open 2021;11:e048690. doi:10.1136/ bmjopen-2021-048690

- Prepublication history and supplemental material for this paper is available online. To view these files, please visit the journal online (http://dx.doi. org/10.1136/bmjopen-2021 048690)

Received 04 January 2021 Accepted 19 May 2021

D) Check for updates

(c) Author(s) (or their employer(s)) 2021. Re-use permitted under CC BY-NC. No commercial re-use. See rights and permissions. Published by BMJ.

For numbered affiliations see end of article.

\section{Correspondence to} Dr Norrisa Adrianna Haynes; norrisa.haynes@pennmedicine. upenn.edu

\section{ABSTRACT}

Background Cardiovascular disease (CVD) remains a leading cause of morbidity and mortality worldwide, conferring a disparate burden on low-income and middleincome countries (LMICs). Haiti represents a resourceconstrained setting, limited by a paucity of resources and trained cardiovascular professionals equipped to address the increasing burden of CVD.

Objective Here, we describe the creation of a comprehensive cardiology curriculum delivered through a virtual classroom. The curriculum was created to augment cardiovascular education in LMICs such as Haiti.

Methods Over one academic year (May 2019-2020), International Cardiology Curriculum Accessible by Remote Distance Learning-Haiti consisted of biweekly, livestreamed, synchronous didactic lectures, seminars and case presentations broadcasted to 16 internal medicine (IM) residents at Hôpital Universitaire de Mirebalais, one of only four IM training programmes in Haiti. The virtual classroom was created using commercially available videoconferencing and data-sharing platforms. Prelecture and postlecture surveys and an end of the year survey were administered to assess the impact of the curriculum. Results Participant performance analysis revealed that $80 \%$ of the curriculum demonstrated a positive trend in knowledge acquisition postintervention. Based on the end of the year evaluation, $94 \%$ of participants reported that the curriculum was educational and relevant to medical practice in Haiti and $100 \%$ reported that the curriculum was good to excellent. Additionally, the curriculum was cited as an effective means of maintaining trainee education during the COVID-19 pandemic.

Conclusion This international medical education pilot study demonstrates the feasibility of augmenting cardiology education in LMICs by creating a virtual curriculum made possible by local partnerships, internet access and technology.

\section{INTRODUCTION}

Cardiovascular disease (CVD) accounts for $29 \%$ of all deaths in Haiti, and the incidence of peripartum cardiomyopathy (PPCM) is one of the highest in the world at a rate of 1 in every 300 deliveries. ${ }^{12}$ Haiti also has a
Strengths and limitations of this study

- The application of the Analysis, Design, Development, Implementation and Evaluation instructional framework enabled a methodical and iterative approach to the development and implementation of an international curriculum.

- Objective knowledge and subjective comfort assessments enabled measurement of knowledge acquisition and improvement in self-reported comfort with the management of cardiovascular patients.

- Partnership among Haitian, American and French health professionals in the design, development and implementation of the curriculum helped to ensure that the curriculum was applicable to providers in low resource settings such as Haiti.

- Given that the initial implementation of the pilot occurred at one training site, the sample size is small.

- The use of immediate postlecture assessments limited the ability to understand program influence on long-term knowledge retention.

relatively high prevalence of rheumatic heart disease (RHD) at 5\% in rural settings and a high prevalence of hypertensive cardiomyopathy and stroke. ${ }^{34}$ There is also a lack of cardiovascular healthcare infrastructure. Although most hospitals have access to ECG machines, there are no catheterisation laboratories in the country. Acute coronary syndromes are typically managed medically. There is limited access to cardiac surgery through non-governmental organisations. Additionally, due to a lack of trained professionals, few hospitals routinely use echocardiography despite its high diagnostic value.

Currently, there are only 16 cardiologists countrywide for a population of over 11 million people. ${ }^{45}$ There are no formal cardiology training programmes in Haiti. The 16 cardiologists who currently work in Haiti received their training outside of the country 
given the absence of domestic postgraduate training programmes in CVD. Many obtained formal cardiology fellowship training in countries such as France, Cuba and the USA. Given the lack of training opportunities and cardiologists in the context of high CVD burden, it is apparent that there is a need for additional cardiovascular education support for healthcare providers both in and out of training programmes.

Hôpital Universitaire de Mirebalais (HUM) is one of only four internal medicine (IM) residency programmes in Haiti, training an estimated $20 \%-25 \%$ of all IM residents in the country. ${ }^{6} \mathrm{HUM}$ is one of the largest teaching hospitals and one of the only to receive accreditation from the Accreditation Council for Graduate Medical Education International-I. ${ }^{7}$ It has an integrated public and private structure with a robust partnership with Haiti's Ministry of Health. Its structure enables it to be both affordable and accessible to the general public. HUM also has access to imaging modalities such as ultrasound making echocardiography possible. Patients travel long distances to seek treatment for some of the most advanced diseases and severe pathology. This holds true for cardiovascular patients with nearly $40 \%$ of all admissions to the IM ward being for heart failure (HF) and $60 \%$ of those HF admissions being women. ${ }^{3}$ Despite the high prevalence of CVD among hospitalised patients at HUM, access to cardiologists is limited. Thus, the IM residents are left to care for these patients without access to optimal cardiovascular education and support.

To assist the cardiovascular needs at HUM, one of the largest teaching hospitals which serves a sizeable catchment area, we developed a virtual pilot educational programme entitled International Cardiology Curriculum Accessible by Remote Distance Learning (ICARDsHaiti) to deliver an adaptive cardiovascular curriculum to IM residents at HUM. Initially developed and executed before the onset of the COVID-19 pandemic, the purpose of ICARDs-Haiti has evolved. The value and goals of the curriculum are now two-fold: to provide pertinent educational material and to maintain educational activities during the ongoing COVID-19 pandemic in Haiti. Here, we describe a pilot intervention and first attempt at bridging the cardiovascular education divide in a resource-limited setting through the execution of a virtual classroom using live-streamed lectures and an interactive discussion format.

\section{Methods}

In May 2019, ICARDs-Haiti was created by a chief resident at HUM (who is now a cardiology trainee in France) and a University of Pennsylvania (UPenn) Cardiology fellow under the auspices of the educational leadership from their respective institutions. Thereafter, a formal international collaboration between HUM physicians and UPenn cardiologists was forged with the objective to create a dynamic, adaptive and comprehensive curriculum that included locally relevant and useful educational cardiology content.
Prior to initiating ICARDs-Haiti, the cardiology curriculum at HUM was undergoing significant restructuring due to the lack of a full-time cardiovascular specialist. The HUM IM faculty created a list of pertinent cardiovascular topics. They planned for oral presentations by residents and a weekly academic discussion with a local cardiologist. Despite these aspirations, in practice, the amount of time that local cardiovascular specialists were able to commit to education at HUM was limited. Additionally, travel restrictions that were imposed due to political instability (and later the COVID-19 pandemic) prohibited travel of visiting cardiologists from the USA and Europe to support trainees and faculty at HUM. The consequence of these limitations was a lack of routine, consistent and comprehensive cardiovascular education despite the high CVD burden among HUM patients. Due to the need for improved and continual cardiovascular education, HUM chief residents and a HUM-affiliated, local cardiologist sought assistance in creating a more consistent and comprehensive cardiology curriculum for HUM's trainees by soliciting assistance from colleagues abroad who had spent significant time in Haiti. A global network of medical educators was subsequently created to address this need.

Through international collaboration, a revised list of pertinent cardiology topics and respective learning objectives was created. The American College of Cardiology core competency recommendations for cardiology trainees were also used as a guide. Volunteer lecturers consisted of cardiology fellows, attendings and internists from UPenn, Boston University, Mount Sinai Medical Centre in Miami, New York University, SUNY Downstate and Centre Hospitalier d'Antibes Juan-les-Pins (Provence Alpes Côtes dAzur, France). Many of the lecturers had personal ties to Haiti and were also multilingual. Lectures were given in either English, French, Haitian Creole or a mixture of these languages. The assessed curricular content was created and delivered by 8 lecturers $50 \%$ of whom were multilingual. The vast majority of IM residents at HUM are fluent in English, French and Haitian Creole, although English comprehension skills are variable. The curriculum was implemented using the Analysis, Design, Development, Implementation, Evaluation training model, an instructional design methodology. ${ }^{8}$ It is currently being used as a guide for the implementation and adaptation of this curriculum based on written and verbal feedback from the IM residents.

Lectures were delivered biweekly via video conference platforms, and each lecture was preceded and followed by anonymous, unmatched preknowledge and postknowledge assessments. The assessments were delivered via the Qualtrics programme and distributed via a commercially available, internet-based mobile phone communication application. Lecture reading materials were distributed via Searchable Log of All Conversation and Knowledge, an online communication platform. The cardiovascular topics and learning objectives for the curriculum were determined by a leadership council 
Table 1 International Cardiology Curriculum Accessible by Remote Distance Learning (ICARDs) 2019-2020

\begin{tabular}{|c|c|c|c|c|}
\hline & Topics & & $\begin{array}{l}\text { Frequency of } \\
\text { sessions }\end{array}$ & $\begin{array}{l}\text { No of } \\
\text { lectures }\end{array}$ \\
\hline \multirow[t]{6}{*}{ Curriculum } & Stenotic valvular lesions, & Congenital heart disease & \multirow[t]{6}{*}{ Biweekly } & \multirow[t]{6}{*}{20} \\
\hline & Heart failure (HF) & Peripheral arterial disease & & \\
\hline & Peripartum cardiomyopathy & Syncope & & \\
\hline & Cardiogenic shock & Hypertension & & \\
\hline & Preoperative workup & Perioperative medication management & & \\
\hline & Interpretation of basic echocardiography & & & \\
\hline \multirow{2}{*}{$\begin{array}{l}\text { Medical/surgical } \\
\text { subcategories }\end{array}$} & \multicolumn{4}{|l|}{ Critical care management } \\
\hline & \multicolumn{4}{|l|}{ Surgical appropriateness } \\
\hline
\end{tabular}

which consisted of cardiovascular fellows and faculty at UPenn as well as the IM programme director and the chief residents at HUM and a local Haitian cardiologist. The content for each lecture was created by volunteer lecturers from the aforementioned institutions and was reviewed by the leadership council. The live-streamed lectures were supplemented with case presentations, seminars and included active audience participation. The cardiology topics covered in the curriculum are shown in table 1. For most of the topics, the following content was provided: physiology, pathophysiology, clinical presentation, physical examination findings, medical management and indications for interventional procedures and surgery.

The ICARDs curriculum was integrated into the trainees' pre-existing didactic schedule. Thus, all 16 residents participated in this 1-year research study. Sessions were held on Monday evenings for 1 hour. Surveys included subjective confidence rating assessments typically with five answer choices that most commonly ranged from strongly disagree to strongly agree. Objective assessments took the form of multiple-choice questions with correct and incorrect answers (online supplemental appendix 1).

\section{Patient and Public Involvement}

Patients or the public were not involved in the design, or conduct, or reporting, or dissemination of this research.

\section{Statistical analysis}

Statistical analysis was performed using SAS software version 9.4. The Wilcoxon Mann-Whitney $\mathrm{U}$ test was used for data analysis due to the non-parametric nature of the data and the small sample size. Cohen's(r)was used to estimate effect sizes. Effect sizes ranging from 0.1 to $<0.3$ were interpreted as small, effect sizes ranging from 0.3 to $<0.5$ were interpreted as moderate, and effect sizes of 0.5 and greater were interpreted as large. ${ }^{9}$

\section{RESULTS}

Between May 2019 and May 2020, there were 16 IM residents at HUM of whom $20 \%$ were women and $80 \%$ were men. There were six first-year residents, five second-year residents and five third-year residents. The ages of the IM residents ranged from 25 to 30 years old. Analysis of 56 items (online supplemental appendix 1) revealed that $32 \%$ of the assessed curricular content was associated with a small effect size, $39 \%$ was associated with a moderate effect size and $29 \%$ was associated with a large effect size.

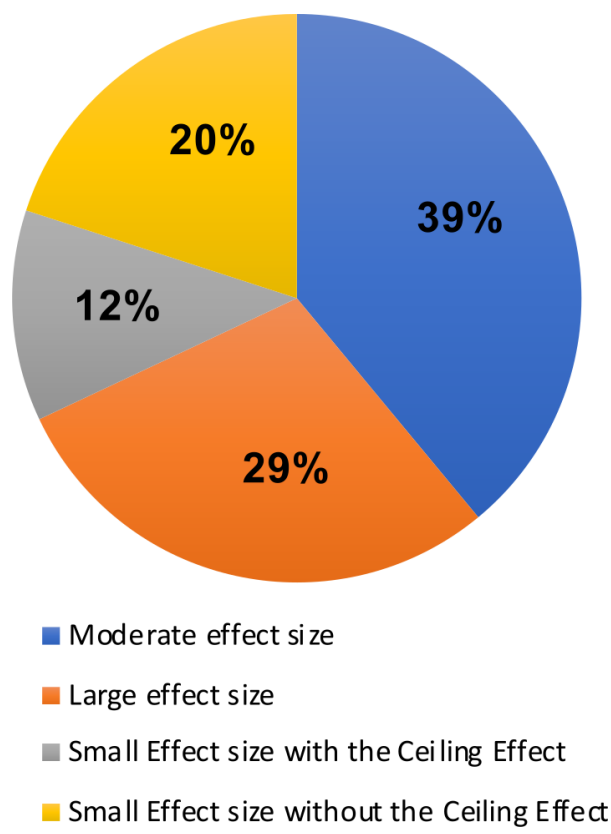

Figure 1 Shows the effect size proportionality for the entire ICARDs-Haiti curriculum. As shown above, $68 \%$ of the curriculum was associated with a moderate to large effect size. Twelve per cent of the curriculum was associated with a small effect size due to the ceiling effect while $20 \%$ of the curriculum was associated with a small effect size not attributable to the ceiling effect. ICARDs, International Cardiology Curriculum Accessible by Remote Distance Learning. 


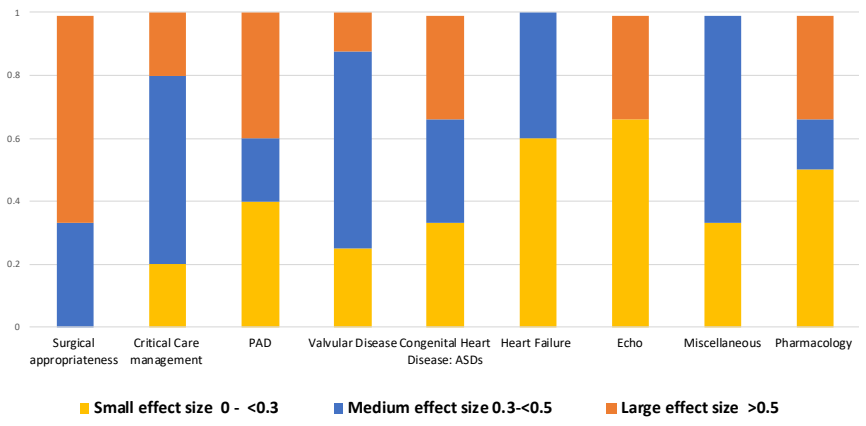

Figure 2 Shows the proportion of topics associated with large, moderate and small effect size. The majority of surgical appropriateness, critical care management, peripheral arterial disease, valvular disease and congenital heart disease were associated with medium to large effect sizes. The miscellaneous category (syncope and COVID-19) had an even split between small and moderate to large effect sizes. The majority of heart failure, echo and pharmacology were associated with small effect sizes with and without the ceiling effect. PAD, peripheral arterial disease.

The small effect size category was further subdivided into a small effect size due to the ceiling effect and a small effect size without the Ceiling Effect. We defined the ceiling effect as subjective or objective assessments associated with a small effect size $<0.3$ for which the postassessments had perfect scores (the ceiling). The percentage of the curriculum with a small effect size with and without the Ceiling Effect was 12\% and 20\%, respectively (figure 1). When the 56 items were analysed based on topic category, we found that the majority of the effect sizes for surgical appropriateness, critical care management, Peripheral arterial disease (PAD), valvular disease and Congenital heart disease (CHD) were moderate to large suggesting that these topics had more of an impact (figure 2). In contrast, HF, echocardiography and pharmacology had a larger proportion of small effect sizes implying that there was no meaningful difference between the preassessment and postassessment (figure 2).

A notable proportion of the small effect sizes was due to the Ceiling Effect with high preassessment scores and postassessment scores of $100 \%$ (online supplemental appendix 2). Additionally, figure 3 shows that topics taught by monolingual English speakers were associated with a higher proportion of small effect sizes without the ceiling effect (24\%) when compared with lectures provided by multilingual lecturers (14\%) shown in figure 4. Four objective assessments; comprehension of the continuity equation, appropriate dosing of dopamine for cardiogenic shock, identifying a hemodynamically significant Qp:Qs ratio (shunt fraction), and correctly identifying all guideline directed medical therapy (GDMT) for HF, were associated with small negative effect sizes, due to a slightly higher proportion of students answering the objective questions correctly prior to the intervention. Notably,

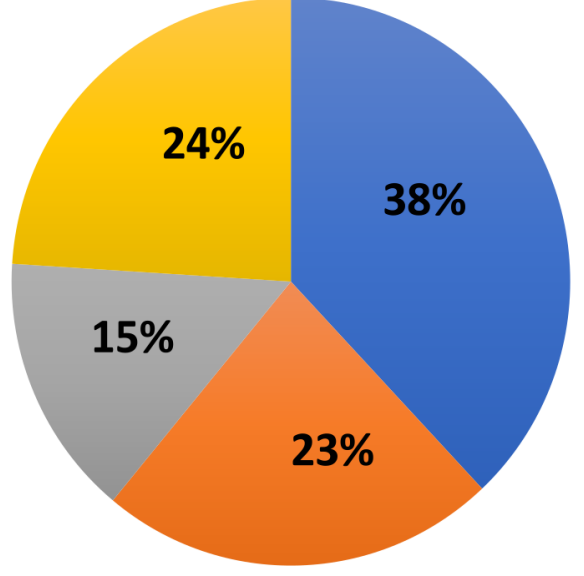

$$
\begin{aligned}
& \text { Moderate effect size } \\
& \text { Large effect size } \\
& \text { Small Effect size with the Cei ling Effect } \\
& \text { Small Effect size without the Ceiling Effect }
\end{aligned}
$$

Figure 3 Shows the association between monolingual English lecturers and effect size. Sixty-one per cent of the topics taught by monolingual English lecturers were associated with moderate to large effect sizes. Twenty-four per cent of the topics taught by monolingual English lecturers were associated with small effect sizes not attributable to the ceiling to effect.

these topics were taught by monolingual English speakers (online supplemental appendix 2).

All participants completed an end-of-the year survey. When asked whether the material covered by the curriculum was relevant to medical practice in Haiti, $56.3 \%$ of participants agreed and $37.5 \%$ strongly agreed for a total of $94 \%$ agreeing that the material was relevant. When asked about the commitment of the course directors, $12.5 \%$ of participants reported the commitment as good and $87.5 \%$ as very good to excellent. In the context of clearly delineated learning objectives, all participants reported learning what they expected from the curriculum with $46 \%$ reporting that the curriculum exceeded their learning expectations. For the overall evaluation of the course, all participants reported that the course was good to excellent. When asked if they would like to incorporate recorded lectures into the curriculum, $25 \%$ of participants reported that they preferred only live lectures, $75 \%$ preferred a mix of live and pre-recorded lectures and $0 \%$ preferred only prerecorded lectures.

When asked how the curriculum could be improved, participants recommended streamlining the lectures, increasing the number of practice questions, incorporating French and Haitian Creole verbal or written translation into the lectures and implementing a hands-on echocardiography experience. Participants identified a number of strengths which included but were not limited to the diversity of lecturers, incorporation of French and Haitian Creole into some of the lectures, the relevance of the content to medical practice in Haiti, the amount of 


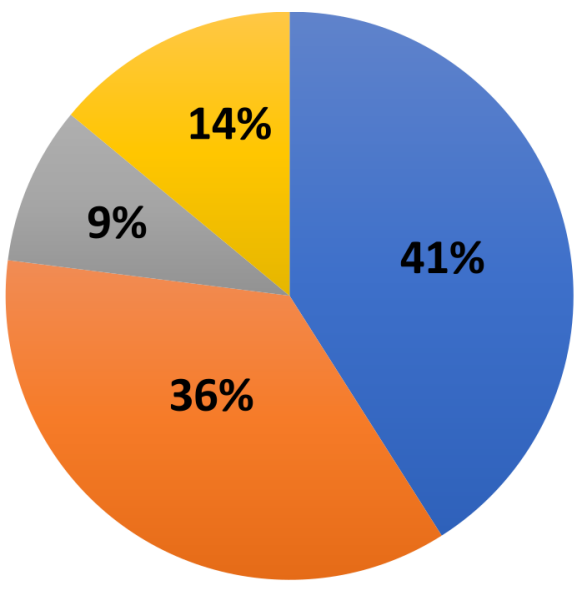

- Moderate effect size

- Large effect size

Small Effect size with the Ceiling Effect

Small Effect size without the Ceiling Effect

Figure 4 Shows the association between multilingual lecturers and effect size. Seventy-seven per cent of the topics taught by multilingual lecturers were associated with medium to large effect sizes. Fourteen per cent of the topics taught by multilingual lecturers were associated with small effect sizes not attributable to the ceiling effect.

material learnt and the prelecture and postlecture questions as pertinent learning aids to reinforce retention of the information presented in the lectures.

\section{DISCUSSION}

In an attempt to address the deficiency of formal cardiovascular education and routine access to cardiologists in Haiti, a virtual, collaborative, international cardiology curriculum for IM trainees was created at HUM, where nearly $40 \%$ of all admissions to the medical ward are due to CVD. ${ }^{3}$ Analysis results suggest that the curriculum has been impactful. Accordingly, $68 \%$ of the assessed curriculum had a moderate to large effect size indicating a noteworthy educational impact based on prelecture and postlecture assessments. When small effect sizes due to the Ceiling Effect are included, 80\% of the curriculum demonstrates a positive trend in knowledge acquisition post-intervention. Furthermore, all participants completed the end of the year general assessment for which the overall rating of the curriculum was good to excellent.

The topics associated with moderate to large effect sizes included surgical appropriateness, critical care management, PAD, CHD and valvular heart disease. After completing the curriculum learning objectives, trainees felt more confident in their ability to identify indications for interventional procedures and surgical intervention for valvular disease and CHD. Trainees were also more confident in knowing when to appropriately refer patients to cardiovascular specialists for corrective interventions. Additionally, trainees demonstrated an improvement in the objective assessments of preoperative risk, medical management of valvular disease and critical care management. An improvement in the assessment scores of the aforementioned topics is important given the high prevalence of RHD and severe HF among patients admitted to HUM. Based on a study by Kwan et $a l, 37 \%$ of all HUM admissions were for HF and more than $60 \%$ of HF patients were women. ${ }^{3}$ Of those women with cardiomyopathy, $50 \%$ were diagnosed with PPCM. ${ }^{3}$ Five percent of cardiovascular admissions were due to RHD with notable valvular disease identified by echocardiography. ${ }^{3}$ Notably, although access to cardiac surgery is limited, organisations such as the Haiti Cardiac Alliance work to facilitate surgical referrals and long-term follow-up for cardiac surgery patients in Haiti. ${ }^{10}$ Thus, educating GPs and internists on timely referral of cardiovascular patients to specialists and entities equipped to provide specialised care, as well as corrective interventions, can improve cardiovascular outcomes for patients with severe CVD in Haiti.

The topics associated with a large proportion of the small effect sizes are shown in figure 2. Many of the small effect sizes were explained by trainees' pre-existing medical knowledge and comfort with those topics. One reason that trainees may have scored highly on a number of the preassessments could be from prior exposure to these topics, before ICARDs-Haiti curriculum implementation. Additionally, trainees were not separated by their training year. Senior residents were likely to be more familiar with some of these topics than junior residents were, thereby, creating smaller effect sizes than if trainees had been separated by their training year. Four topics (shunt fraction, continuity equation comprehension, HF GDMT and Dopamine dosing) were associated with small negative effect sizes. All four of these assessments were objective and were taught only in English without French or Haitian Creole translation. Thus, the lack of a positive and moderate to large effect size for these topics is likely multifactorial and causality may include a lack of content clarity, topic complexity and language comprehension. A larger proportion of topics taught by multilingual lecturers with personal ties to Haiti, were associated with moderate to large effect sizes. Studies have demonstrated the effect of culture and language on online learning including how linguistic barriers and academic culture can negatively impact the academic success of non-English speakers. ${ }^{11} 12$ This observation highlights the importance of not only language, but also cultural contextualisation for learning.

Specific considerations were made to ensure that the curriculum was applicable to providers practicing in Haiti. Health professionals from Haiti participated in the design, development and implementation of the curriculum. These collaborations enabled an understanding of specific resource limitations that were taken into account when creating the curriculum. The inclusion and active participation of Haitian health professionals 
reinforced culturally responsive teaching, which likely mitigated cultural barriers. ${ }^{13}$ Their participation enabled a real-world application of the curriculum that helped to fill an educational void that hopefully also enhanced peer-to-peer education. Additionally, the prevalence of various CVDs in Haiti was used to prioritise topics. Lastly, feedback from the residents was obtained to improve the quality and relevance of the curriculum content.

Looking ahead, there are plans to modify the ICARDs curriculum to better address the educational needs in this region. Written or verbal French or Haitian Creole translation will be provided for all future lectures. For the topics associated with small effect sizes without the Ceiling Effect, these lectures will be restructured to improve clarity. For the topics associated with small effect sizes with the ceiling effect (implying that the trainees were already familiar with the topics), there will be a refocus of educational efforts on other higher yield topics. Learning objectives for various topics will be stratified by training year. To improve the objective assessment results, homework assignments which will be reviewed in smaller interactive summer sessions to support comprehension of complex topics. Drumford and Miller, measured increased student engagement from collaborative team and seminar modalities in online learning, highlighting their import in the acquisition of group skills and needful student-faculty interactions. ${ }^{14}$ To diversify the mode of curriculum delivery, we will include both synchronous and asynchronous educational content, modelling the flipped classroom approach which, when done correctly, has been proven to be an effective teaching methodology. ${ }^{15-17}$ Additionally, other quantitatively proven methods for augmented remote learning such as discussion boards, chat rooms and interactive guided problem solving are being explored for integration into the curriculum. ${ }^{14}$ As per participant feedback, hands-on experiences using diagnostic tools for capacity building and skill development will be implemented. Additionally, residents will be followed longitudinally for 3 years into their first year of independent practice as internists to assess the longitudinal impact of the curriculum.

This pilot study was a success given the establishment of an international collaboration that succeeded in creating a dynamic curriculum that augmented cardiovascular education for IM trainees in Haiti. To improve the curriculum, the aforementioned changes will be made, and longitudinal impact will be measured. If there is a marked improvement, ICARDs will be expanded to additional sites to deliver much needed cardiovascular education to general practitioners, mid-level providers and internists in low-resource settings.

\section{Limitations}

The current study is the first to describe and present data in support of the utilisation of an adaptive international cardiology curriculum delivered via an interactive and dynamic virtual classroom in Haiti. However, the study has specific limitations. The small sample size is a limitation. Given the grassroots nature of ICARDs-Haiti, it was initially launched at one teaching hospital as a pilot programme which limited the sample size. Additionally, only immediate preassessment and postassessment results were obtained. Thus, although knowledge acquisition was notable among the residents at HUM, the impact on long-term knowledge retention remains unclear. Additionally, language differences were associated with lower assessment performance. This is supported by the fact that topics taught by multilingual lecturers were more likely to be associated with moderate to large effect sizes. The fact that trainees were not separated by their training year is another limitation of the study and likely led to smaller effect sizes. Lastly, although lecture materials were reviewed by the leadership council and although the diversity of the lecturer cohort was a strength of the curriculum, variability in lecture delivery and verbal communication may have impacted the effect size for certain topics.

\section{CONCLUSION}

The alarming increase in the global burden of CVD provides compelling evidence in support of prioritising urgent, yet, carefully planned efforts to support cardiovascular medical education in LMICs such as Haiti. By using a live, dynamic and virtual classroom, this international cardiology curriculum demonstrated positive trends in knowledge acquisition through pre, post and end of the year assessments. This accomplishment is noteworthy given the grassroots effort among Haitian, American and French cardiology trainees and faculty to support cardiovascular educational capacity in one of the most under-resourced countries in the world despite cultural and socioeconomic barriers. Future directions of this experience will lend themselves towards improving the curriculum and standardising a component of the lecture platform. This will allow for both synchronous and asynchronous educational development that can eventually be expanded to other resource-limited settings that lack cardiology training programmes and specialists. In the long run, the hope is that this will help to address global health disparities in cardiovascular education in resourcelimited settings and serve as a valuable adjunct to onsite global health endeavours in the future.

\section{Author affiliations}

${ }^{1}$ Cardiology, University of Pennsylvania, Philadelphia, Pennsylvania, USA

${ }^{2}$ Cardiology, Centre Hospitalier d Antibes Juan les Pins, Antibes, Provence-AlpesCôte d'Azu, France

${ }^{3}$ Cardiovascular Surgery, University of Pennsylvania, Philadelphia, Pennsylvania, USA

${ }^{4}$ University of Pennsylvania Perelman School of Medicine, Philadelphia, Pennsylvania, USA

${ }^{5}$ Internal Medicine, Hôpital Universitaire de Mirebalais, Mirebalais, Haiti

${ }^{6}$ Center for Clinical Epidemiology and Biostatistics, University of Pennsylvania, Philadelphia, Pennsylvania, USA

${ }^{7}$ Division of General Internal Medicine, University of Pennsylvania, Philadelphia, Pennsylvania, USA 


\section{Twitter Norrisa Adrianna Haynes @NorrisaHaynes}

Acknowledgements Special thank you to the internal medicine residents at Hôpital Universitaire de Mirebalais, Dr. Jean-Pierre Brisma, Zanmi Lasante, Dr. Merly Robert, Dr. Gene Kwan, UPenn cardiology fellows, Dr. Glenn Gaulton, Penn Center for Global Health, Dr. Garly Saint-Croix, Dr. Michel Ibrahim, Dr. Benedicte LeFevre, Dr. Yann Dagher-Hayck, Dr. Anne Christine Buteau, Dr. Krystal Hill, Dr. Paul Mather, Dr. Victor Ferrari, Dr. Mahesh Vidula, Dr. Sri Adusumalli, Dr. Thomas Cappola, Dr. Frank Silvestry, Denice Susini, Jennifer Cronin, and funding from the Penn Cardiovascular Disease Fellowship Innovation Fund.

Contributors NAH and VS-J conceived and planned the study. NAH, VS-J, FVV, CD, DL and MSA carried out the study. NAH and VS-J performed data collection. AT, NAH, VS-J, JS, MSA and JAS performed data analysis and interpretation. NAH, JS and VS-J drafted the manuscript. MSA, JAS and AE performed critical revision of the article. NAH, VS-J, JS, AE, FVV, CD, DL, AT, JAS and MSA approved the article to be published.

Funding The authors have not declared a specific grant for this research from any funding agency in the public, commercial or not-for-profit sectors.

Competing interests None declared.

Patient consent for publication Not required.

Ethics approval IRB exemptions were obtained from the Zanmi Lasante/HUM and UPenn IRB committees.

Provenance and peer review Not commissioned; externally peer reviewed.

Data availability statement All data relevant to the study are included in the article or uploaded as online supplemental information.

Supplemental material This content has been supplied by the author(s). It has not been vetted by BMJ Publishing Group Limited (BMJ) and may not have been peer-reviewed. Any opinions or recommendations discussed are solely those of the author(s) and are not endorsed by BMJ. BMJ disclaims all liability and responsibility arising from any reliance placed on the content. Where the content includes any translated material, BMJ does not warrant the accuracy and reliability of the translations (including but not limited to local regulations, clinical guidelines, terminology, drug names and drug dosages), and is not responsible for any error and/or omissions arising from translation and adaptation or otherwise.

Open access This is an open access article distributed in accordance with the Creative Commons Attribution Non Commercial (CC BY-NC 4.0) license, which permits others to distribute, remix, adapt, build upon this work noncommercially, and license their derivative works on different terms, provided the original work is properly cited, appropriate credit is given, any changes made indicated, and the use is non-commercial. See: http://creativecommons.org/ licenses/by-nc/4.0/.

\section{ORCID iD}

Norrisa Adrianna Haynes http://orcid.org/0000-0001-8028-7133

\section{REFERENCES}

1 WHO | Noncommunicable diseases country profiles, 2018WHO. Available: https://www.who.int/nmh/countries/en/ [Accessed 11 Nov 2020].

2 Davis MB, Arany Z, McNamara DM, et al. Peripartum cardiomyopathy: JACC state-of-the-art review. J Am Coll Cardiol 2020;75:207-21.

3 Kwan GF, Jean-Baptiste W, Cleophat P, et al. Descriptive epidemiology and short-term outcomes of heart failure hospitalisation in rural Haiti. Heart 2016;102:140-6.

4 synthèse EVIRde. | UNICEF Haïti, 2018. Available: https://www. unicef.org/haiti/rapports/emmus-vi-rapport-de-synth\%C3\%A8se2018 [Accessed 25 Aug 2020].

5 Le Nouvelliste - Lueur d'espoir pour la cardiologie en Haïti. Available: https://lenouvelliste.com/article/104570/lueur-despoir-pour-lacardiologie-en-haiti [Accessed 6 Sep 2020].

6 Ministère de la Santé Publique et de la population. Available: https:// mspp.gouv.ht/newsite/documentation.php?page=362\&param1 = valu1\&param2=value2 [Accessed 7 Sep 2020].

7 University hospital in Haiti Earns global accreditation as teaching institution | partners in health. Available: https://www.pih.org/article/ university-hospital-haiti-earns-global-accreditation-teachinginstitution [Accessed 5 Nov 2020].

8 Patel SR, Margolies PJ, Covell NH, et al. Using instructional design, analyze, design, develop, implement, and evaluate, to develop e-learning modules to Disseminate supported employment for community behavioral health treatment programs in New York state. Front Public Health 2018;6:113.

9 Fritz CO, Morris PE, Richler JJ. Effect size estimates: current use, calculations, and interpretation. J Exp Psychol Gen 2012;141:2-18.

10 Robinson O, Kwan GF, Romain J-L, et al. A national coordinated cardiac surgery registry in Haiti: the Haiti cardiac alliance experience. Lancet Glob Health 2016;4:S31.

11 Kang, Haijun, Mason R, et al. Opportunity and e-quality: Intercultural and linguistic issues in global online learning. Distance Education 2014.

12 Goodfellow R, Lea M, Gonzalez F, et al. Opportunity and e-quality: Intercultural and linguistic issues in global online learning. Distance Education 2001;22:65-84.

13 Gay G. Preparing for culturally responsive teaching. J Teach Educ 2002;53:106-16.

14 Dumford AD, Miller AL. Online learning in higher education: exploring advantages and disadvantages for engagement. $J$ Comput High Educ 2018;30:452-65.

15 Narang A, Velagapudi P, Rajagopalan B, et al. A New Educational Framework to Improve Lifelong Learning for Cardiologists. J Am Coll Cardiol 2018;71:454-62.

16 Burke AS, Fedorek B. Does "flipping" promote engagement?: A comparison of a traditional, online, and flipped class. Active Learning in Higher Education 2017;18:11-24.

17 Lin H-C, Hwang G-J. Research trends of flipped classroom studies for medical courses: a review of Journal publications from 2008 to 2017 based on the technology-enhanced learning model. Interactive Learning Environments 2018:1-17. 